\title{
Approaches to Multiple Attribute Decision-Making with Fuzzy Number Intuitionistic Fuzzy Information and Their Application to English Teaching Quality Evaluation
}

\author{
Zhen Zhang $\mathbb{B}^{1}$ and Pengfei Su $\mathbb{i}^{2}$ \\ ${ }^{1}$ School of Foreign Language Studies, Xi'an Aeronautical University, Xi'an 710077, Shaanxi, China \\ ${ }^{2}$ Modern Chemistry Research Institute, Xi'an 710065 Shaanxi, China \\ Correspondence should be addressed to Zhen Zhang; janezhen@xaau.edu.cn and Pengfei Su; 95601943@qq.com
}

Received 11 July 2021; Accepted 26 November 2021; Published 31 December 2021

Academic Editor: Juan L. G. Guirao

Copyright ( 2021 Zhen Zhang and Pengfei Su. This is an open access article distributed under the Creative Commons Attribution License, which permits unrestricted use, distribution, and reproduction in any medium, provided the original work is properly cited.

\begin{abstract}
Many experts and scholars focus on the Maclaurin symmetric mean (MSM) operator, which can reflect the interrelationship among the multi-input arguments. It has been generalized to different fuzzy environments and put into use in various actual decision problems. The fuzzy number intuitionistic fuzzy numbers (FNIFNs) could well depict the uncertainties and fuzziness during the English teaching quality evaluation. And the English teaching quality evaluation is frequently viewed as the multiple attribute decision-making (MADM) issue. We expand the MSM equation with FNIFNs to propose the fuzzy number intuitionistic fuzzy MSM (FNIFMSM) equation and fuzzy number intuitionistic fuzzy weighted MSM (FNIFWMSM) equation in this study. A few MADM tools are developed with FNIFWMSM equation. Finally, taking English teaching quality evaluation as an example, this paper illustrates the depicted approach.
\end{abstract}

\section{Introduction}

In 1965, Zadeh [1] established a novel fuzzy set (FS) to deal with decision information in the fuzzy new domain [2-7]. To extend novel FS, the intuitionistic fuzzy sets (IFSs) $[8,9]$ were developed. Subsequently, FS and its related extension knowledges are exploited into the more and more decision domains [10-17]. Iakovidis and Papageorgiou [18] defined the cognitive maps for medical decision making under IFSs. $\mathrm{Li}$ [19] built the GOWA operator to MADM using IFSs. Su et al. [20] proposed the interactive method for dynamic IFMAGDM. Tan [21] constructed the Choquet integral-based TOPSIS method for IF-MADM. Wu and Zhang [22] built the IF-MADM based on weighted entropy. Yu [23] defined the generalized prioritized geometric operators under IFSs. Yu et al. [24] defined the derivatives and differentials for multiplicative IFSs. Zhao et al. [25] defined the interactive intuitionistic fuzzy algorithms for multilevel programming problems. Arya and Yadav [26] defined the intuitionistic fuzzy super-efficiency slack-based measure. Büyüközkan et al. [27] selected the transportation schemes with the integrated intuitionistic fuzzy Choquet integral method. De and Sana [28] defined the $(p, q, r, l)$ method for random demand with Bonferroni mean under IFSs. Garg [29] proposed the improved cosine similarity measure for IFSs. Joshi et al. [17] defined the Jensen-alpha-Norm dissimilarity measure for IFSs. Li et al. [30] defined the time-preference and VIKOR-based dynamic method for IF-MADM. The authors in [31] built the intuitionistic fuzzy MABAC method based on cumulative prospect theory for MAGDM. Niroomand [32] defined the multiobjective-based direct solution method for linear programming along with intuitionistic fuzzy parameters. Zhao et al. [33] perfected TODIM for IF-MAGDM on the strength of cumulative prospect theory. Furthermore, Liu and Yuan [34] built the fuzzy number IFSs (FNIFSs) to combine the IFSs with the triangular fuzzy sets (TFSs). Li et al. [35] developed the entropy and similarity measure under FNIFSs. Wang [36] built the geometric operators under FNIFSs. Verma [37] defined the GFNIFWBM operator under FNIFSs. 
Nevertheless, all the functions and tools proposed by the above scholars do not take into account the relationship between parameters [38-41]. To conquer these shortcomings, the crucial purpose of the article is to connect the FNIFSs with MSM operator [42-47] to build several novel fused formulas under FNIFSs.

Consequently, the rest work would be depicted. Several basic concepts of FNIFSs and MSM formulas would be depicted in the second section. The MSM formulas with FNIFSs would be constructed in the third section. An instance about English teaching quality evaluation is given in the fourth section. The conclusions reached will be depicted the last section.

\section{Preliminaries}

In this section, we introduced the concept of fuzzy number intuitionistic fuzzy sets (FNIFSs) [32] and the Maclaurin symmetric mean (MSM) operator [44].

2.1. Fuzzy Number Intuitionistic Fuzzy Set. Liu and Yuan [34] gave the definition of FNIFS, and the membership and nonmembership are given in the form of TFNs.

Definition 1 (see [34]). Supposed $E=\left\{e_{1}, e_{2}, \ldots, e_{n}\right\}$ is a fixed set, $B$ is a FNIFS on $E$ and its expression form is given as follows:

$$
B=\epsilon\left\{\left\langle e, T_{B}(e), F_{B}(e)\right\rangle e \in E\right\} .
$$

$T_{B}(e)$ and $F_{B}(e)$ are two TFNs between 0 and 1 , and $T_{B}(e)=(X(e), Y(e), Z(e)), e \longrightarrow[0,1], \quad F_{B}(e)=(A(e)$, $S(e), D(e)), E \longrightarrow[0,1]$, and $0 \leq Z(e)+D(e) \leq 1, \forall e \in E$.
Let $\quad T_{B}(e)=(X(e), Y(e), Z(e)), \quad F_{B}(e)=(A(e)$, $S(e), D(e)), \quad$ so $\quad Q(e)=\langle(X(e), Y(e), Z(e))$, $(A(e), S(e), D(e))\rangle, Q(e)$ is viewed as a FNIFN.

Definition 2 (see [36, 48]). $Q\left(e_{i}\right)=\left\langle\left(X\left(e_{i}\right), Y\left(e_{i}\right)\right.\right.$, $\left.\left.Z\left(e_{i}\right)\right),\left(A\left(e_{i}\right), S\left(e_{i}\right), D\left(e_{i}\right)\right)\right\rangle$ and $Q\left(e_{j}\right)=\left\langle\left(X\left(e_{j}\right), Y\left(e_{j}\right)\right.\right.$, $\left.\left.Z\left(e_{j}\right)\right),\left(A\left(e_{j}\right), S\left(e_{j}\right), D\left(e_{j}\right)\right)\right\rangle$ are two FNIFNs.

$$
\begin{aligned}
& \text { (1) } Q\left(e_{i}\right) \oplus Q\left(e_{j}\right)=\quad\left\{\left(\begin{array}{c}
X\left(e_{i}\right)+X\left(e_{j}\right)-X\left(e_{i}\right) X\left(e_{j}\right), \\
Y\left(e_{i}\right)+Y\left(e_{j}\right)-Y\left(e_{i}\right) Y\left(e_{j}\right), \\
Z\left(e_{i}\right)+Z\left(e_{j}\right)-Z\left(e_{i}\right) Z\left(e_{j}\right)
\end{array}\right)\right. \text {, } \\
& \left.\left(\begin{array}{c}
A\left(e_{i}\right) A\left(e_{j}\right) \\
S\left(e_{i}\right) S\left(e_{j}\right) \\
D\left(e_{i}\right) D\left(e_{j}\right)
\end{array}\right)\right\}
\end{aligned}
$$

(2) $Q\left(e_{i}\right) \otimes Q\left(e_{j}\right)=$

$$
\left\{\left(\left(\begin{array}{c}
X\left(e_{i}\right) X\left(e_{j}\right) \\
Y\left(e_{i}\right) Y\left(e_{j}\right), \\
Z\left(e_{i}\right) Z\left(e_{j}\right)
\end{array}\right)\right)\right.
$$$$
\left.\left(\left(\begin{array}{c}
A\left(e_{i}\right)+A\left(e_{j}\right)-A\left(e_{i}\right) A\left(e_{j}\right), \\
S\left(e_{i}\right)+S\left(e_{j}\right)-S\left(e_{i}\right) S\left(e_{j}\right), \\
D\left(e_{i}\right)+D\left(e_{j}\right)-D\left(e_{i}\right) D\left(e_{j}\right)
\end{array}\right)\right)\right\}
$$

(3) $\lambda Q\left(e_{i}\right)=\left\{\left(\begin{array}{c}1-\left(1-X\left(e_{i}\right)\right)^{\lambda}, \\ 1-\left(1-Y\left(e_{i}\right)\right)^{\lambda}, \\ 1-\left(1-Z\left(e_{i}\right)\right)^{\lambda}\end{array}\right),\left(\begin{array}{c}\left(A\left(e_{i}\right)\right)^{\lambda}, \\ \left(S\left(e_{i}\right)\right)^{\lambda}, \\ \left(D\left(e_{i}\right)\right)^{\lambda}\end{array}\right)\right\}$,

(4) $\left(Q\left(e_{i}\right)\right)^{\lambda}=\left\{\left(\begin{array}{c}\left(X\left(e_{i}\right)\right)^{\lambda}, \\ \left(Y\left(e_{i}\right)\right)^{\lambda}, \\ \left(Z\left(e_{i}\right)\right)^{\lambda}\end{array}\right),\left(\begin{array}{c}1-\left(1-A\left(e_{i}\right)\right)^{\lambda}, \\ 1-\left(1-S\left(e_{i}\right)\right)^{\lambda}, \\ 1-\left(1-D\left(e_{i}\right)\right)^{\lambda}\end{array}\right)\right\}$, $\lambda \geq 0$

Definition 3 (see $[36,48])$. Let $Q(e)=\langle(X(e), Y(e)$, $Z(e)),(A(e), S(e), D(e))\rangle$ be a given FNIFN, a score function of a FNIFN $Q(e)$ can be depicted as follows:

$$
\operatorname{SF}(Q(e))=\frac{X(e)+2 Y(e)+Z(e)}{4}-\frac{A(e)+2 S(e)+D(e)}{4}, \quad \operatorname{SF}(Q(e)) \in[-1,1]
$$

Definition 4 (see [36, 48]). Let $Q(e)=\langle(X(e)$, $Y(e), Z(e)),(A(e), S(e), D(e))\rangle$ be a given FNIFN, an accuracy function of a FNIFN $Q(e)$ can be defined as follows:

$$
\begin{array}{r}
\mathrm{AH}(\mathrm{Q}(e))=\frac{X(e)+2 Y(e)+Z(e)}{4}+\frac{A(e)+2 S(e)+D(e)}{4}, \\
\operatorname{AH}(Q(e)) \in[-1,1]
\end{array}
$$

Based on the $\operatorname{SF}(Q(e))$ and $\operatorname{AH}(Q(e))$, next, let us look at the size comparison of the two FNIFNs.

Definition 5 (see $[36,48]$ ). Let $Q\left(e_{1}\right)$ and $Q\left(e_{2}\right)$ be two FNIFNs, then if $\operatorname{SF}\left(Q\left(e_{1}\right)\right)<\operatorname{SF}\left(Q\left(e_{2}\right)\right)$, then $Q\left(e_{1}\right)<Q\left(e_{2}\right)$; if $\operatorname{SF}\left(Q\left(e_{1}\right)\right)=\operatorname{SF}\left(Q\left(e_{2}\right)\right)$, then

(1) If $\mathrm{AH}\left(\mathrm{Q}\left(e_{1}\right)\right)=\mathrm{AH}\left(\mathrm{Q}\left(e_{2}\right)\right)$, then $\mathrm{Q}\left(e_{1}\right)=\mathrm{Q}\left(e_{2}\right)$

(2) If $\mathrm{AH}\left(\mathrm{Q}\left(e_{1}\right)\right)<\mathrm{AH}\left(\mathrm{Q}\left(e_{2}\right)\right)$, then $\mathrm{Q}\left(e_{1}\right)<\mathrm{Q}\left(e_{2}\right)$
2.2. MSM Operators. Maclaurin [44] proposed the MSM formula.

Definition 6 (see [44]). Let $g_{m}(m=1,2, \ldots, k)$ be is a real number greater than 0 , and $n=(1,2, \ldots, k)$. If

$\operatorname{MSM}^{(n)}\left(g_{1}, g_{2}, \ldots, g_{k}\right)=\left(\sum_{1 \leq m_{1}<\cdots<m_{n} \leq m_{k}} \frac{\prod_{l=1}^{n} c_{m_{l}}}{C_{k}^{n}}\right)^{(1 / n)}$,

then we call $\operatorname{MSM}^{(n)}$ the MSM formula, where $\left(m_{1}, m_{2}, \ldots, m_{n}\right)$ traverses all the k-tuple combinations of $(1,2, \ldots, n)$ and $C_{n}^{k}$ is the binomial coefficient.

\section{FNIFMSM and FNIFWMSM Operators}

3.1. The FNIFMSM Operator. Here, we are going to expand MSM to coalesce all FNIFNs and establish the fuzzy number intuitionistic fuzzy MSM (FNIFMSM) operators. 
Definition 7. Let $Q\left(e_{m}\right)=\left\langle\left(X\left(e_{m}\right), Y\left(e_{m}\right), Z\left(e_{m}\right)\right)\right.$, $\left.\left(A\left(e_{m}\right), S\left(e_{m}\right), D\left(e_{m}\right)\right)\right\rangle, m=1,2, \ldots, k$, be a set of given
FNIFNs. The FNIFMSM operator could be depicted as follows:

$$
\operatorname{FNIFMSM}^{(n)}\left(Q\left(e_{1}\right), Q\left(e_{2}\right), \ldots, Q\left(e_{k}\right)\right)=\left(\frac{\oplus_{1 \leq m_{1}<\ldots<m_{n} \leq m_{k}}\left(\otimes_{l=1}^{n} Z\left(x_{m_{l}}\right)\right)}{C_{k}^{n}}\right)^{(1 / n)}
$$

Theorem 1. $Q\left(e_{m}\right)=\left\langle\left(X\left(e_{m}\right), \quad Y\left(e_{m}\right), Z\left(e_{m}\right)\right),\left(A\left(e_{m}\right), \quad\right.\right.$ The coalesced data obtained from FNIFMSM equations are $\left.\left.S\left(e_{m}\right), D\left(e_{m}\right)\right)\right\rangle, m=1,2, \ldots, k$, be a suite of given FNIFNs. still an FNIFN.

$$
\begin{aligned}
& \operatorname{FNIFMSM}^{(n)}\left(Q\left(e_{1}\right), Q\left(e_{2}\right), \ldots, Q\left(e_{k}\right)\right)=\left(\frac{\oplus_{1 \leq m_{1}<\cdots<m_{n} \leq m_{k}}\left(\otimes_{l=1}^{n} Q\left(e_{m_{l}}\right)\right)}{C_{k}^{n}}\right)^{(1 / n)}
\end{aligned}
$$

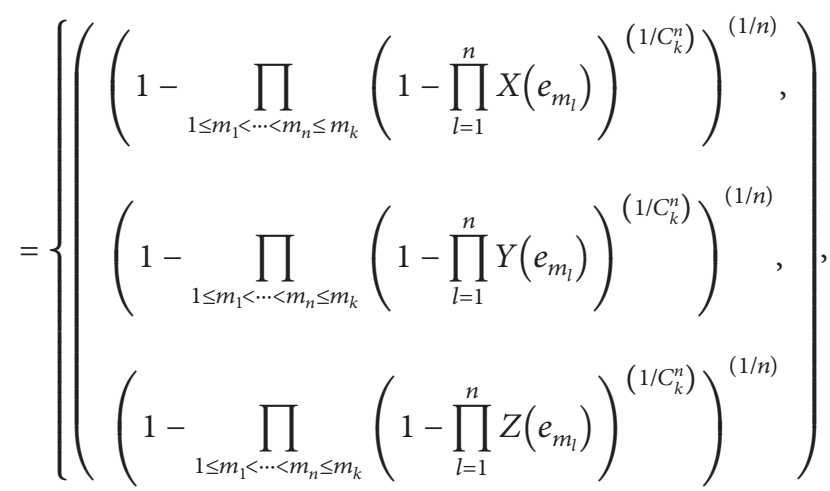

$$
\begin{aligned}
& \left.\left\{\begin{array}{l}
1-\left(1-\prod_{1 \leq m_{1}<\cdots<m_{n} \leq m_{k}}\left(1-\prod_{l=1}^{n}\left(1-A\left(e_{m_{j}}\right)\right)\right)^{\left(1 / C_{k}^{n}\right)}\right)^{(1 / n)}, \\
1-\left(1-\prod_{1 \leq m_{1}<\cdots<m_{n} \leq m_{k}}\left(1-\prod_{l=1}^{n}\left(1-S\left(e_{m_{j}}\right)\right)\right)^{\left(1 / C_{k}^{n}\right)}\right)^{(1 / n)}, \\
1-\left(1-\prod_{1 \leq m_{1}<\cdots<m_{n} \leq m_{k}}\left(1-\prod_{l=1}^{n}\left(1-D\left(e_{m_{j}}\right)\right)\right)^{\left(1 / C_{k}^{n}\right)}\right)^{(1 / n)}
\end{array}\right)\right\} .
\end{aligned}
$$

Proof. According to Definition 2, we can derive

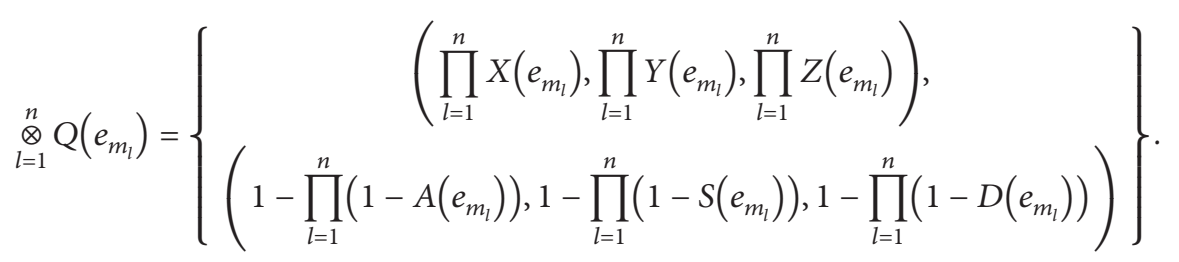

Thus, 
4

Discrete Dynamics in Nature and Society

$$
\begin{aligned}
& \underset{1 \leq m_{1}<\cdots<m_{n} \leq m_{k}}{\oplus}\left(\stackrel{n}{\otimes} Q\left(e_{m_{l}}\right)\right) \\
& =\left\{\left(\begin{array}{c}
1-\prod_{1 \leq m_{1}<\cdots<m_{n} \leq m_{k}}\left(1-\prod_{l=1}^{n} X\left(e_{m_{l}}\right)\right) \\
1-\prod_{1 \leq m_{1}<\cdots<m_{n} \leq m_{k}}\left(1-\prod_{l=1}^{n} Y\left(e_{m_{l}}\right)\right) \\
1-\prod_{1 \leq m_{1}<\cdots<m_{n} \leq m_{k}}\left(1-\prod_{l=1}^{n} Z\left(e_{m_{l}}\right)\right)
\end{array}\right),\left(\begin{array}{r}
\prod_{1 \leq m_{1}<\cdots<m_{n} \leq m_{k}}\left(1-\prod_{l=1}^{n}\left(1-A\left(e_{m_{l}}\right)\right)\right), \\
\prod_{1 \leq m_{1}<\cdots<m_{n} \leq m_{k}}\left(1-\prod_{l=1}^{n}\left(1-S\left(e_{m_{l}}\right)\right)\right), \\
\prod_{1 \leq m_{1}<\cdots<m_{n} \leq m_{k}}\left(1-\prod_{l=1}^{n}\left(1-D\left(e_{m_{l}}\right)\right)\right)
\end{array}\right)\right\} .
\end{aligned}
$$

Thereafter,

$$
\begin{aligned}
& \frac{\oplus_{1 \leq m_{1}<\cdots<m_{n} \leq m_{k}}\left(\otimes_{j=1}^{k} Q\left(e_{m_{l}}\right)\right)}{C_{n}^{k}}
\end{aligned}
$$

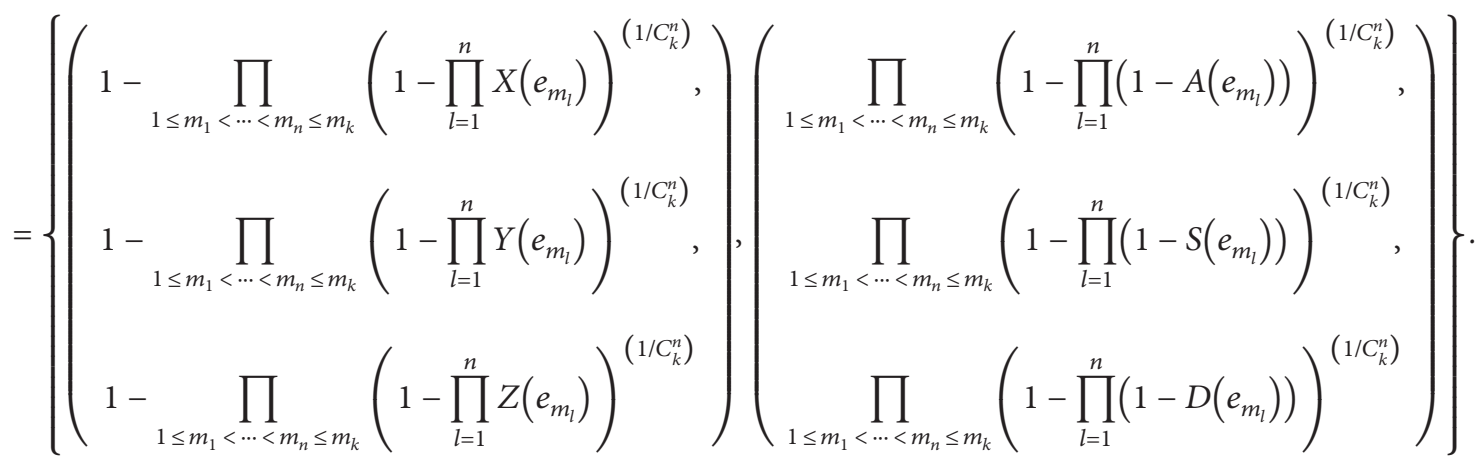

Therefore, 


$$
\begin{aligned}
& \operatorname{FNIFMSM}^{(n)}\left(Q\left(e_{1}\right), Q\left(e_{2}\right), \ldots, Q\left(e_{k}\right)\right)=\left(\frac{\oplus_{1 \leq m_{1}<\cdots<m_{n} \leq m_{k}}\left(\otimes_{l=1}^{n} Q\left(e_{m_{l}}\right)\right)}{C_{k}^{n}}\right)^{(1 / n)}
\end{aligned}
$$

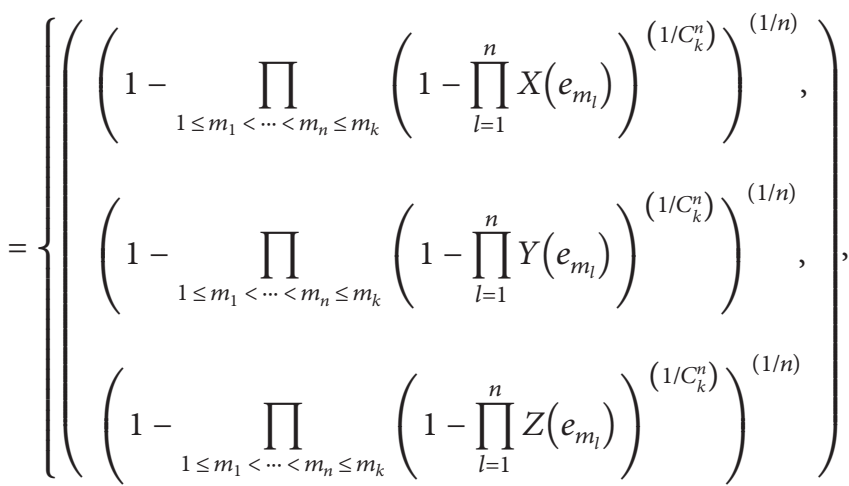

$$
\begin{aligned}
& \left.\left\{\begin{array}{c}
1-\left(1-\prod_{1 \leq m_{1}<\cdots<m_{n} \leq m_{k}}\left(1-\prod_{l=1}^{n}\left(1-A\left(e_{m_{l}}\right)\right)\right)^{\left(1 / C_{k}^{n}\right)}\right)^{(1 / n)} \\
1-\left(1-\prod_{1 \leq m_{1}<\cdots<m_{n} \leq m_{k}}\left(1-\prod_{l=1}^{n}\left(1-S\left(e_{m_{l}}\right)\right)\right)^{\left(1 / C_{k}^{n}\right)}\right)^{(1 / n)} \\
1-\left(1-\prod_{1 \leq m_{1}<\cdots<m_{n} \leq m_{k}}\left(1-\prod_{l=1}^{n}\left(1-D\left(e_{m_{l}}\right)\right)\right)^{\left(1 / C_{k}^{n}\right)}\right)^{(1 / n)}
\end{array}\right)\right\} \\
& 0 \leq \prod_{1 \leq m_{1}<\ldots<m_{n} \leq m_{k}}\left(1-\prod_{l=1}^{n} X\left(e_{m_{l}}\right)\right)^{\left(1 / C_{k}^{n}\right)} \leq 1
\end{aligned}
$$
FNIFN. We need to prove two following conditions:

(1) $(X(e), Y(e), Z(e)) \subseteq[0,1],(A(e), S(e), D(e)) \subseteq[0,1]$

(2) $0 \leq Z(e)+D(e) \leq 1$

Proof. (1) Since $0 \leq X\left(e_{m_{l}}\right) \leq 1$, we get

$$
\begin{aligned}
& 0 \leq \prod_{l=1}^{n} X\left(e_{m_{l}}\right) \leq 1, \\
& 0 \leq\left(1-\prod_{l=1}^{n} X\left(e_{m_{l}}\right)\right)^{\left(1 / C_{k}^{n}\right)} \leq 1 .
\end{aligned}
$$

Then,
Thus,

$0 \leq\left(1-\prod_{1 \leq m_{1}<\cdots<m_{n} \leq m_{k}}\left(1-\prod_{l=1}^{n} X\left(e_{m_{l}}\right)\right)^{\left(1 / C_{k}^{n}\right)}\right)^{(1 / n)} \leq 1$.

That is to say, $X(e)=\left(1-\prod_{1 \leq m_{1}<\cdots<m_{n} \leq m_{k}}\right.$ (1$\left.\left.\prod_{l=1}^{n} X\left(e_{m_{l}}\right)\right)^{\left(1 / C_{k}^{n}\right)}\right)^{(1 / n)} \subseteq[0,1]$; similarly, we can get $(Y(e), Z(e)) \subseteq[0,1]$ and $(A(e), S(e), D(e)) \subseteq[0,1]$, so (1) is maintained.

(2) For $Z\left(e_{m_{l}}\right)+D\left(e_{m_{l}}\right) \leq 1$, we can derive $Z\left(e_{m_{l}}\right) \leq 1-D\left(e_{m_{l}}\right)$; thus, 


$$
\begin{aligned}
0 & \leq Z(e)+D(e) \\
& =\left(1-\prod_{1 \leq m_{1}<\cdots<m_{n} \leq m_{k}}\left(1-\prod_{l=1}^{n} X\left(e_{m_{l}}\right)\right)^{\left(1 / C_{k}^{n}\right)}\right)^{(1 / n)}+1-\left(1-\prod_{1 \leq m_{1}<\cdots<m_{n} \leq m_{k}}\left(1-\prod_{l=1}^{n} D\left(e_{m_{l}}\right)\right)^{\left(1 / C_{k}^{n}\right)}\right)^{(1 / k)} \\
& \leq\left(1-\prod_{1 \leq m_{1}<\cdots<m_{n} \leq m_{k}}\left(1-\prod_{l=1}^{n} D\left(e_{m_{l}}\right)\right)^{\left(1 / C_{k}^{n}\right)}\right)^{(1 / n)}+1-\left(1-\prod_{1 \leq m_{1}<\cdots<m_{n} \leq m_{k}}\left(1-\prod_{l=1}^{n} D\left(e_{m_{l}}\right)\right)^{\left(1 / C_{k}^{n}\right)}\right)^{(1 / k)} \\
& =1 .
\end{aligned}
$$

Example 1. Let $Q\left(e_{1}\right)=\langle(0.1,0.2,0.3),(0.2,0.5,0.6)\rangle$, $Q\left(e_{2}\right)=\langle(0.2,0.3,0.3),(0.2,0.5,0.5)\rangle$, and $Q\left(e_{3}\right)=\langle(0.4$, $0.5,0.5),(0.3,0.4,0.4)\rangle$ be three FNIFNs and suppose $n=2$, then according to (6), we have

$$
\begin{aligned}
& \operatorname{FNIFMSM}^{(n)}\left(Q\left(e_{1}\right), Q\left(e_{2}\right), \ldots, Q\left(e_{k}\right)\right)=\left(\frac{\oplus_{1 \leq m_{1}<\cdots<m_{n} \leq m_{k}}\left(\otimes_{l=1}^{n} Q\left(e_{m_{l}}\right)\right)}{C_{k}^{n}}\right)^{(1 / n)} \\
& \left\{\begin{array}{c}
\left(\begin{array}{l}
\left(1-((1-0.1 \times 0.2) \times(1-0.1 \times 0.4) \times(1-0.2 \times 0.4))^{\left(1 / C_{3}^{2}\right)}\right)^{(1 / 2)}, \\
\left(1-((1-0.2 \times 0.3) \times(1-0.2 \times 0.5) \times(1-0.3 \times 0.5))^{\left(1 / C_{3}^{2}\right)}\right)^{(1 / 2)}, \\
\left(1-((1-0.3 \times 0.3) \times(1-0.3 \times 0.5) \times(1-0.3 \times 0.5))^{\left(1 / C_{3}^{2}\right)}\right)^{(1 / 2)}
\end{array}\right), \\
\left(\begin{array}{l}
1-\left(1-((1-(1-0.2) \times(1-0.2)) \times(1-(1-0.2) \times(1-0.3)) \times(1-(1-0.2) \times(1-0.3)))^{\left(1 / C_{3}^{2}\right)}\right)^{(1 / 2)}, \\
1-\left(1-((1-(1-0.5) \times(1-0.5)) \times(1-(1-0.5) \times(1-0.4)) \times(1-(1-0.5) \times(1-0.4)))^{\left(1 / C_{3}^{2}\right)}\right.
\end{array}\right), \\
1-\left(1-((1-(1-0.6) \times(1-0.5)) \times(1-(1-0.6) \times(1-0.4)) \times(1-(1-0.5) \times(1-0.4)))^{\left(1 / C_{3}^{2}\right)}\right.
\end{array}\right\} \\
& =\langle(0.2168,0.3227,0.3612),(0.2329,0.4674,0.5023)\rangle .
\end{aligned}
$$

Next, we explore some properties about the FNIFMSM formula.

Property 1 (idempotency). If $Q\left(e_{m}\right)=\left\langle\left(X\left(e_{m}\right), Y\left(e_{m}\right)\right.\right.$, $\left.\left.Z\left(e_{m}\right)\right),\left(A\left(e_{m}\right), S\left(e_{m}\right), D\left(e_{m}\right)\right)\right\rangle, m=1,2, \ldots, k$ are equal, then

$$
\operatorname{FNIFMSM}^{(n)}\left(Q\left(e_{1}\right), Q\left(e_{2}\right), \ldots, Q\left(e_{k}\right)\right)=Q(e) .
$$

Property 2 (monotonicity). Let $Q\left(e_{m}\right)=\left\langle\left(X\left(e_{m}\right), Y\left(e_{m}\right)\right.\right.$, $\left.\left.Z\left(e_{m}\right)\right),\left(A\left(e_{m}\right), S\left(e_{m}\right), D\left(e_{m}\right)\right)\right\rangle, m=1,2, \ldots, k, \quad$ and $Q\left(f_{m}\right)=\left\langle\left(X\left(f_{m}\right), Y\left(f_{m}\right), Z\left(f_{m}\right)\right), \quad\left(A\left(f_{m}\right), S\left(f_{m}\right)\right.\right.$, $\left.\left.D\left(f_{m}\right)\right)\right\rangle, m=1,2, \ldots, k$, be two sets of given FNIFNs. If $X\left(e_{m}\right) \leq X\left(f_{m}\right), Y\left(e_{m}\right) \leq Y\left(f_{m}\right), Z\left(e_{m}\right) \leq Z\left(f_{m}\right), \quad A\left(e_{m}\right) \geq$ $A\left(f_{m}\right), S\left(e_{m}\right) \geq S\left(f_{m}\right)$, and $D\left(e_{m}\right) \geq D\left(f_{m}\right)$ hold for all $m$, then

$$
\begin{aligned}
& \operatorname{FNIFMSM}^{(n)}\left(Q\left(e_{1}\right), Q\left(e_{2}\right), \ldots, Q\left(e_{k}\right)\right) \\
& \quad \leq \operatorname{FNIFMSM}^{(n)}\left(Q\left(f_{1}\right), Q\left(f_{2}\right), \ldots, Q\left(f_{k}\right)\right) .
\end{aligned}
$$

Property 3 (boundedness). Let $Q\left(e_{m}\right)=\left\langle\left(X\left(e_{m}\right)\right.\right.$, $\left.\left.Y\left(e_{m}\right), Z\left(e_{m}\right)\right),\left(A\left(e_{m}\right), S\left(e_{m}\right), D\left(e_{m}\right)\right)\right\rangle, m=1,2, \ldots, k$, be a set of given FNIFNs. If $Q(e)^{+}=$ $\left\{\begin{array}{c}\left(\max \left(X\left(e_{m}\right)\right), \max \left(Y\left(e_{m}\right)\right), \max \left(Z\left(e_{m}\right)\right)\right), \\ \left(\min \left(A\left(e_{m}\right)\right), \min \left(S\left(e_{m}\right)\right), \min \left(D\left(e_{m}\right)\right)\right)\end{array}\right\}(m=1,2$, $\ldots, k)$ and $Q(e)^{-}=\left\{\left(\min \left(X\left(e_{m}\right)\right), \quad \min \left(Y\left(e_{m}\right)\right)\right.\right.$, $\left.\left.\min \left(Z\left(e_{m}\right)\right)\right),\left(\max \left(A\left(e_{m}\right)\right), \max \left(S\left(e_{m}\right)\right), \quad \max \left(D\left(e_{m}\right)\right)\right)\right\}$ $(m=1,2, \ldots, k)$, then

$$
Q(e)^{-} \leq \operatorname{FNIFMSM}^{(n)}\left(Q\left(e_{q}\right), Q\left(e_{2}\right), \ldots, Q\left(e_{n}\right)\right) \leq Q(e)^{+} .
$$


Property 4 (commutativity). Let $Q\left(e_{m}\right)(m=1,2, \ldots, k)$ be a set of given FNIFNs and $Q\left(e_{m}^{\prime}\right)(m=1,2, \ldots, k)$ be any permutation of $Q\left(e_{m}\right)(m=1,2, \ldots, k)$, then

$$
\begin{aligned}
& \operatorname{FNIFMSM}^{(n)}\left(Q\left(e_{1}\right), Q\left(e_{2}\right), \ldots, Q\left(e_{m}\right)\right) \\
& \quad=\operatorname{FNIFMSM}^{(n)}\left(Q\left(e_{1}^{\prime}\right), Q\left(e_{2}^{\prime}\right), \ldots,\left(e_{m}^{\prime}\right)\right) .
\end{aligned}
$$

3.2. The FNIFWMSM Operator. In real-life MADM, it is crucial to fully take attribute weights into account. We shall build the fuzzy number intuitionistic fuzzy weighted MSM (FNIFWMSM) formula.

Definition 8 . Let $Q\left(e_{m}\right)=\left\langle\left(X\left(e_{m}\right), Y\left(e_{m}\right), Z\left(e_{m}\right)\right)\right.$, $\left.\left(A\left(e_{m}\right), S\left(e_{m}\right), D\left(e_{m}\right)\right)\right\rangle(m=1,2, \ldots, k)$ be a set of given FNIFNs with weight vector $\xi_{m}=\left(\xi_{1}, \xi_{2}, \ldots, \xi_{k}\right)^{T}$ and $\xi_{m} \in[0,1], \sum_{m=1}^{k} \xi_{m}=1$. If

$$
\operatorname{FNIFWMSM}_{k \xi}^{(n)}\left(Q\left(e_{1}\right), Q\left(e_{2}\right), \ldots, Q\left(e_{k}\right)\right)
$$

$$
=\left(\frac{\oplus_{1 \leq m_{1}<\cdots<m_{n} \leq m_{k}}\left(\otimes_{l=1}^{n}\left(k \xi_{m_{l}} \otimes Q\left(e_{m_{l}}\right)\right)\right)}{C_{k}^{n}}\right)^{(1 / n)},
$$

then we called FNIFWMSM ${ }_{k \xi}^{(n)}$ the fuzzy number intuitionistic fuzzy weighted MSM (FNIFWMSM) formula.

Theorem 2. Let $Q\left(e_{m}\right)=\left\langle\left(X\left(e_{m}\right), Y\left(e_{m}\right), Z\left(e_{m}\right)\right)\right.$, $\left.\left(A\left(e_{m}\right), S\left(e_{m}\right), D\left(e_{m}\right)\right)\right\rangle(i=1,2, \ldots, n)$ be a set of given FNIFNs. The coalesced data obtained from the FNIFWMSM formula are still a FNIFN.

$$
\begin{aligned}
& \operatorname{FNIFWMSM}_{k \xi}^{(n)}\left(Q\left(e_{1}\right), Q\left(e_{2}\right), \ldots, Q\left(e_{k}\right)\right)=\left(\frac{\oplus_{1 \leq m_{1}<\cdots<m_{n} \leq m_{k}}\left(\otimes_{l=1}^{n}\left(k \xi_{m_{l}} \otimes Z\left(e_{m_{l}}\right)\right)\right)}{C_{k}^{n}}\right)^{(1 / n)} \\
& =\left\{\begin{array}{l}
\left(\begin{array}{l}
\left(1-\prod_{1 \leq m_{1}<\cdots<m_{n} \leq m_{k}}\left(1-\prod_{l=1}^{n}\left(1-\left(1-X\left(e_{m_{l}}\right)\right)^{k \xi_{m_{l}}}\right)\right)^{\left(1 / C_{k}^{n}\right)}\right)^{(1 / n)} \\
\left(1-\prod_{1 \leq m_{1}<\cdots<m_{n} \leq m_{k}}\left(1-\prod_{l=1}^{n}\left(1-\left(1-Y\left(e_{m_{l}}\right)\right)^{k \xi_{m_{l}}}\right)\right)^{\left(1 / C_{k}^{n}\right)}\right)^{(1 / n)} \\
\left(1-\prod_{1 \leq m_{1}<\cdots<m_{n} \leq m_{k}}\left(1-\prod_{l=1}^{n}\left(1-\left(1-Z\left(e_{m_{l}}\right)\right)^{k \xi_{m_{l}}}\right)\right)^{\left(1 / C_{k}^{n}\right)}\right)^{(1 / n)}
\end{array}\right.
\end{array}\right. \\
& \left.\begin{array}{l}
1-\left(1-\prod_{1 \leq m_{1}<\ldots<m_{n} \leq m_{k}}\left(1-\prod_{l=1}^{n}\left(1-\left(A\left(e_{m_{l}}\right)\right)^{k \xi_{m_{l}}}\right)\right)^{\left(1 / C_{k}^{n}\right)}\right)^{(1 / n)} \\
1-\left(1-\prod_{1 \leq m_{1}<\ldots<m_{n} \leq m_{k}}\left(1-\prod_{l=1}^{n}\left(1-\left(S\left(e_{m_{l}}\right)\right)^{k \xi_{m_{l}}}\right)\right)^{\left(1 / C_{k}^{n}\right)}\right)^{(1 / n)} \\
1-\left(1-\prod_{1 \leq m_{1}<\cdots<m_{n} \leq m_{k}}\left(1-\prod_{l=1}^{n}\left(1-\left(D\left(e_{m_{l}}\right)\right)^{k \xi_{m_{l}}}\right)\right)^{\left(1 / C_{k}^{n}\right)}\right)^{(1 / n)}
\end{array}\right\} .
\end{aligned}
$$


8

Discrete Dynamics in Nature and Society

Proof. According to Definition 2, we could derive

$$
k \xi_{m_{l}} \otimes Z\left(e_{m_{l}}\right)=\left\{\begin{array}{c}
\left(1-\left(1-X\left(e_{m_{l}}\right)\right)^{k \xi_{m_{l}}}, 1-\left(1-Y\left(e_{m_{l}}\right)\right)^{k \xi_{m_{l}}}, 1-\left(1-Z\left(e_{m_{l}}\right)\right)^{k \xi_{m_{l}}}\right), \\
\left(\left(A\left(e_{m_{l}}\right)\right)^{\xi_{m_{l}}},\left(S\left(e_{m_{l}}\right)\right)^{\xi_{m_{l}}},\left(D\left(e_{m_{l}}\right)\right)^{\xi_{m_{l}}}\right)
\end{array}\right\} .
$$

Thus,

$$
\begin{aligned}
& \underset{l=1}{\stackrel{n}{\otimes}}\left(k \xi_{m_{l}} \otimes Q\left(e_{m_{l}}\right)\right) \\
& \quad=\left\{\begin{array}{l}
\left(\prod_{l=1}^{n}\left(1-\left(1-X\left(e_{m_{l}}\right)\right)^{k \xi_{m_{l}}}\right), \prod_{l=1}^{n}\left(1-\left(1-Y\left(e_{m_{l}}\right)\right)^{k \xi_{m_{l}}}\right), \prod_{l=1}^{n}\left(1-\left(1-Z\left(e_{m_{l}}\right)\right)^{k \xi_{m_{l}}}\right)\right) \\
\left(1-\prod_{l=1}^{n}\left(1-\left(A\left(e_{m_{l}}\right)\right)^{k \xi_{m_{l}}}\right), 1-\prod_{l=1}^{n}\left(1-\left(S\left(e_{m_{l}}\right)\right)^{k \xi_{m_{l}}}\right), 1-\prod_{l=1}^{n}\left(1-\left(D\left(e_{m_{l}}\right)\right)^{k \xi_{m_{l}}}\right)\right)
\end{array}\right\} .
\end{aligned}
$$

Thereafter,

$$
\begin{aligned}
& \underset{1 \leq m_{1}<\cdots<m_{n} \leq m_{k}}{\oplus}\left(\underset{l=1}{\otimes}\left(k \xi_{m_{l}} \otimes Q\left(e_{m_{l}}\right)\right)\right) \\
& =\left\{\left(\begin{array}{r}
1-\prod_{1 \leq m_{1}<\ldots<m_{n} \leq m_{k}}\left(1-\prod_{l=1}^{n}\left(1-\left(1-X\left(e_{m_{l}}\right)\right)^{k \xi_{m_{l}}}\right)\right), \\
1-\prod_{1 \leq m_{1}<\cdots<m_{n} \leq m_{k}}\left(1-\prod_{l=1}^{n}\left(1-\left(1-Y\left(e_{m_{l}}\right)\right)^{k \xi_{m_{l}}}\right)\right), \\
1-\prod_{1 \leq m_{1}<\cdots<m_{n} \leq m_{k}}\left(1-\prod_{l=1}^{n}\left(1-\left(1-Z\left(e_{m_{l}}\right)\right)^{k \xi_{m_{l}}}\right)\right)
\end{array}\right),\left(\begin{array}{r}
\prod_{1 \leq m_{1}<\cdots<m_{n} \leq m_{k}}\left(1-\prod_{l=1}^{n}\left(1-\left(A\left(e_{m_{l}}\right)\right)^{k \xi_{m_{l}}}\right)\right), \\
\prod_{1 \leq m_{1}<\cdots<m_{n} \leq m_{k}}\left(1-\prod_{l=1}^{n}\left(1-\left(S\left(e_{m_{l}}\right)\right)^{k \xi_{m_{l}}}\right)\right) \\
\prod_{1 \leq m_{1}<\ldots<m_{n} \leq m_{k}}\left(1-\prod_{l=1}^{n}\left(1-\left(D\left(e_{m_{l}}\right)\right)^{k \xi_{m_{l}}}\right)\right)
\end{array}\right)\right\} .
\end{aligned}
$$

Furthermore,

$$
\begin{aligned}
& \frac{\oplus_{1 \leq m_{1}<\cdots<m_{n} \leq m_{k}}\left(\otimes_{l=1}^{n}\left(k \xi_{m_{l}} \otimes Q\left(e_{m_{l}}\right)\right)\right)}{C_{k}^{n}} \\
& =\left\{\begin{array}{l}
\left.1-\prod_{1 \leq m_{1}<\cdots<m_{n} \leq m_{k}}\left(1-\prod_{l=1}^{n}\left(1-\left(1-X\left(e_{m_{l}}\right)\right)^{k \xi_{m_{l}}}\right)\right)^{\left(1 / C_{k}^{n}\right)},\right) \\
1-\prod_{1 \leq m_{1}<\cdots<m_{n} \leq m_{k}}\left(1-\prod_{l=1}^{n}\left(1-\left(1-Y\left(e_{m_{l}}\right)\right)^{k \xi_{m_{l}}}\right)\right)^{\left(1 / C_{k}^{n}\right)}, \\
1-\prod_{1 \leq m_{1}<\cdots<m_{n} \leq m_{k}}\left(1-\prod_{l=1}^{n}\left(1-\left(1-Z\left(e_{m_{l}}\right)\right)^{k \xi_{m_{l}}}\right)\right)^{\left(1 / C_{k}^{n}\right)}
\end{array}\right),\left(\begin{array}{c}
\prod_{1 \leq m_{1}<\cdots<m_{n} \leq m_{k}}\left(1-\prod_{l=1}^{n}\left(1-\left(A\left(e_{m_{l}}\right)\right)^{k \xi_{m_{l}}}\right)\right)^{\left(1 / C_{k}^{n}\right)}, \\
\prod_{1 \leq m_{1}<\cdots<m_{n} \leq m_{k}}\left(1-\prod_{l=1}^{n}\left(1-\left(S\left(e_{m_{l}}\right)\right)^{k \xi_{m_{l}}}\right)\right)^{\left(1 / C_{k}^{n}\right)}, \\
\prod_{1 \leq m_{1}<\cdots<m_{n} \leq m_{k}}\left(1-\prod_{l=1}^{n}\left(1-\left(D\left(e_{m_{l}}\right)\right)^{k \xi_{m_{l}}}\right)\right)^{\left(1 / C_{k}^{n}\right)}
\end{array}\right) .
\end{aligned}
$$


Therefore,

$$
\begin{aligned}
& \operatorname{FNIFWMSM}_{k \xi}^{(n)}\left(Q\left(e_{1}\right), Q\left(e_{2}\right), \ldots, Q\left(e_{k}\right)\right)=\left(\frac{\oplus_{1 \leq m_{1}<\cdots<m_{n} \leq m_{k}}\left(\otimes_{l=1}^{n}\left(k \xi_{m_{l}} \otimes Q\left(e_{m_{l}}\right)\right)\right)}{C_{k}^{n}}\right)^{(1 / n)}
\end{aligned}
$$

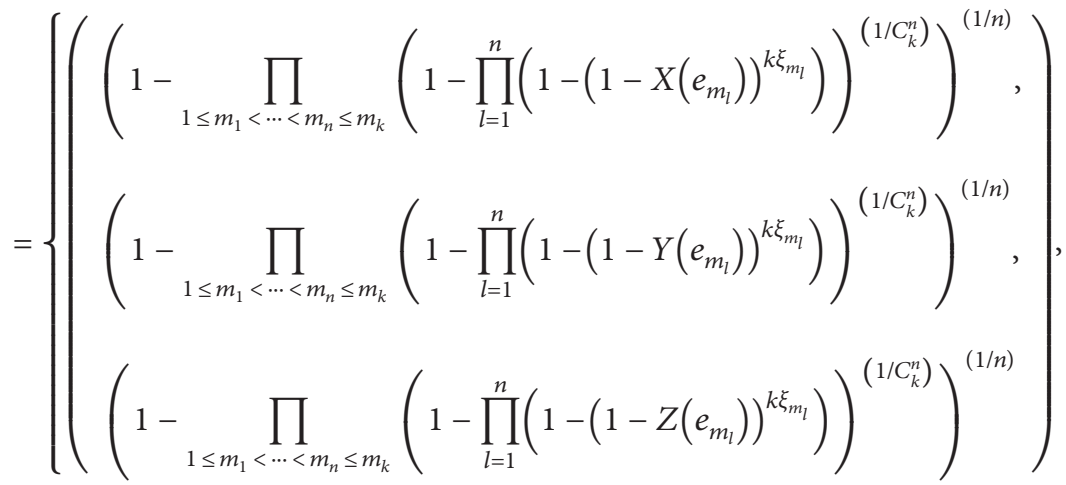

$$
\begin{aligned}
& \left.\left.\begin{array}{l}
1-\left(1-\prod_{1 \leq m_{1}<\ldots<m_{n} \leq m_{k}}\left(1-\prod_{l=1}^{n}\left(1-\left(A\left(e_{m_{l}}\right)\right)^{k \xi_{m_{l}}}\right)\right)^{\left(1 / C_{k}^{n}\right)}\right)^{(1 / n)} \\
1-\left(1-\prod_{1 \leq m_{1}<\cdots<m_{n} \leq m_{k}}\left(1-\prod_{l=1}^{n}\left(1-\left(S\left(e_{m_{l}}\right)\right)^{k \xi_{m_{l}}}\right)\right)^{\left(1 / C_{k}^{n}\right)}\right)^{(1 / n)}
\end{array}\right]\right\} .
\end{aligned}
$$

Hence, (21) is kept.

Then, we could prove that equation (21) is an FNIFN. We need to prove two following conditions:

(1) $(X(e), Y(e), Z(e)) \subseteq[0,1],(A(e), S(e), D(e)) \subseteq[0,1]$

(2) $0 \leq Z(e)+D(e) \leq 1$

Proof. (1) Since $0 \leq X\left(e_{m_{l}}\right) \leq 1$, we get

$$
\begin{aligned}
& 0 \leq\left(1-X\left(e_{m_{l}}\right)\right)^{k \xi_{m_{l}}} \leq 1, \\
& 0 \leq \prod_{l=1}^{n}\left(1-\left(1-X\left(e_{m_{l}}\right)\right)^{k \xi_{m_{l}}}\right) \leq 1 .
\end{aligned}
$$

Then,

$$
0 \leq \prod_{1 \leq m_{1}<\cdots<m_{n} \leq m_{k}}\left(1-\prod_{l=1}^{n}\left(1-\left(1-X\left(e_{m_{l}}\right)\right)^{k \xi_{m_{l}}}\right)\right)^{\left(1 / C_{k}^{n}\right)} \leq 1 .
$$

Thus,

$$
0 \leq\left(1-\prod_{1 \leq m_{1}<\ldots<m_{n} \leq m_{k}}\left(1-\prod_{l=1}^{n}\left(1-\left(1-X\left(e_{m_{l}}\right)\right)^{k \xi_{m_{l}}}\right)\right)^{\left(1 / C_{k}^{n}\right)}\right)^{(1 / n)} \leq 1
$$

That means $\quad X(e)=\left(1-\prod_{1 \leq m_{1}<\cdots<m_{n} \leq}\right.$ $m_{k}\left(1-\prod_{l=1}^{n}\left(1-\left(1-X\left(e_{m_{l}}\right)\right)^{\left.k \xi_{m_{l}}\right)}\right)^{\left(1 / C_{k}^{n}\right)}\right)^{(1 / n)} \subseteq[0,1]$; similarly, we can get $(Y(e), Z(e)) \subseteq[0,1]$, and $(A(e), S(e), D(e)) \subseteq[0,1]$, so (1) is maintained. 
(2) For $Z\left(e_{m_{l}}\right)+D\left(e_{m_{l}}\right) \leq 1$, we can derive $D\left(e_{m_{l}}\right) \leq 1-Z\left(e_{m_{l}}\right)$; thus,

$$
\begin{aligned}
0 \leq Z(e)+D(e)= & \left.\left(1-\prod_{1 \leq m_{1}<\cdots<m_{n} \leq m_{k}}\left(1-\prod_{l=1}^{n}\left(1-\left(1-Z\left(e_{m_{l}}\right)\right)^{k \xi_{m_{l}}}\right)\right)\right)^{\left(1 / C_{k}^{n}\right)}\right)^{(1 / n)} \\
& \left.+1-\left(1-\prod_{1 \leq m_{1}<\cdots<m_{n} \leq m_{k}}\left(1-\prod_{l=1}^{n}\left(1-\left(D\left(e_{m_{l}}\right)\right)^{k \xi_{m_{l}}}\right)\right)\right)^{\left(1 / C_{k}^{n}\right)}\right)^{(1 / k)} \\
\leq & \left(1-\prod_{1 \leq m_{1}<\cdots<m_{n} \leq m_{k}}\left(1-\prod_{l=1}^{n}\left(1-\left(1-Z\left(e_{m_{l}}\right)\right)^{k \xi_{m_{l}}}\right)\right)^{\left(1 / C_{k}^{n}\right)}\right)^{(1 / n)} \\
& +1-\left(1-\prod_{1 \leq m_{1}<\cdots<m_{n} \leq m_{k}}\left(1-\prod_{l=1}^{n}\left(1-\left(1-Z\left(e_{m_{l}}\right)\right)^{k \xi_{m_{l}}}\right)\right)^{\left(1 / C_{k}^{n}\right)}\right)^{(1 / n)}=1 .
\end{aligned}
$$

Example 2. Let $Q\left(e_{1}\right)=\langle(0.1,0.2,0.3),(0.2,0.5,0.6)\rangle$, $\mathrm{Q}\left(e_{2}\right)=\langle(0.2,0.3,0.3),(0.2,0.5,0.5)\rangle$, and $\mathrm{Q}\left(e_{3}\right)=\langle(0.4$, $0.5,0.5),(0.3,0.4,0.4)\rangle$ be three given FNIFNs and suppose $n=2$ and $\xi=(0.2,0.3,0.5)$, then according to $(21)$, we have

$\operatorname{FNIFWMSM}_{k \xi}^{(n)}\left(Q\left(e_{1}\right), Q\left(e_{2}\right), \ldots, Q\left(e_{k}\right)\right)=\left(\frac{\oplus_{1 \leq m_{1}<\cdots<m_{n} \leq m_{k}}\left(\otimes_{l=1}^{n}\left(k \xi_{m_{l}} \otimes Q\left(e_{m_{l}}\right)\right)\right)}{C_{k}^{n}}\right)^{(1 / n)}$

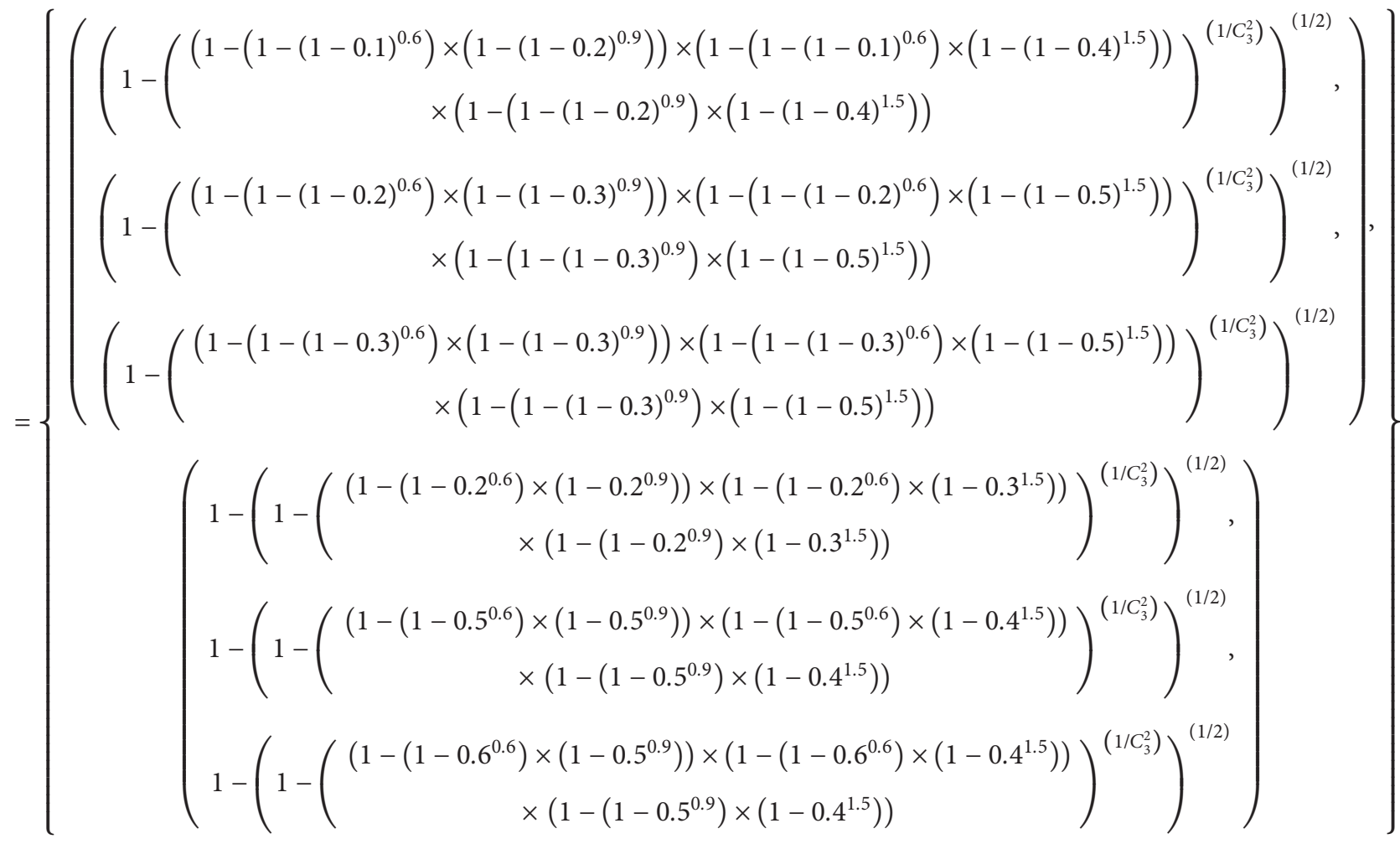

$=\langle(0.2187,0.3157,0.3462),(0.2589,0.4932,0.5226)\rangle$. 
Then, we will discuss some properties of FNIFWMSM operator.

Property 5 (idempotency). If $Q\left(e_{m}\right)=\left\langle\left(X\left(e_{m}\right), Y\left(e_{m}\right)\right.\right.$, $\left.\left.Z\left(e_{m}\right)\right),\left(A\left(e_{m}\right), S\left(e_{m}\right), D\left(e_{m}\right)\right)\right\rangle(m=1,2, \ldots, k)$ are equal, then

$$
\operatorname{FNIFWMSM}_{k \xi}^{(n)}\left(Q\left(e_{1}\right), Q\left(e_{2}\right), \ldots, Q\left(e_{k}\right)\right)=Q(e) .
$$

Property 6 (monotonicity). Let $Q\left(e_{m}\right)=\left\langle\left(X\left(e_{m}\right), Y\left(e_{m}\right)\right.\right.$, $\left.\left.Z\left(e_{m}\right)\right),\left(A\left(e_{m}\right), S\left(e_{m}\right), D\left(e_{m}\right)\right)\right\rangle, m=1,2, \ldots, k, \quad$ and $Q\left(f_{m}\right)=\left\langle\left(X\left(f_{m}\right), Y\left(f_{m}\right), \quad Z\left(f_{m}\right)\right),\left(A\left(f_{m}\right), S\left(f_{m}\right)\right.\right.$, $\left.\left.D\left(f_{m}\right)\right)\right\rangle, m=1,2, \ldots, k$, be two sets of given FNIFNs. If $X\left(e_{m}\right) \leq X\left(f_{m}\right), Y\left(e_{m}\right) \leq Y\left(f_{m}\right), Z\left(e_{m}\right) \leq Z\left(f_{m}\right), \quad A\left(e_{m}\right) \geq$ $A\left(f_{m}\right), S\left(e_{m}\right) \geq S\left(f_{m}\right), D\left(e_{m}\right) \geq D\left(f_{m}\right)$ hold for all $m$, then

$$
\begin{aligned}
& \operatorname{FNIFWMSM}_{k \xi}^{(n)}\left(Q\left(e_{1}\right), Q\left(e_{2}\right), \ldots, Q\left(e_{k}\right)\right) \\
& \quad \leq \operatorname{FNIFWMSM}_{k \xi}^{(n)}\left(Q\left(f_{1}\right), Q\left(f_{2}\right), \ldots, Q\left(f_{k}\right)\right) .
\end{aligned}
$$

Property 7 (boundedness). Let $Q\left(e_{m}\right)=\left\langle\left(X\left(e_{m}\right), Y\left(e_{m}\right)\right.\right.$, $\left.\left.Z\left(e_{m}\right)\right),\left(A\left(e_{m}\right), S\left(e_{m}\right), D\left(e_{m}\right)\right)\right\rangle, m=1,2, \ldots, k$, be a set of given FNIFNs. If $Q(e)^{+}=$ $\left\{\begin{array}{c}\left(\max \left(X\left(e_{m}\right)\right), \max \left(Y\left(e_{m}\right)\right), \max \left(Z\left(e_{m}\right)\right)\right), \\ \left(\min \left(A\left(e_{m}\right)\right), \min \left(S\left(e_{m}\right)\right), \min \left(D\left(e_{m}\right)\right)\right)\end{array}\right\} \quad(m=1,2$ $, \ldots, k)$ and $Q(e)^{-}=\left\{\begin{array}{l}\left(\min \left(X\left(e_{m}\right)\right), \min \left(Y\left(e_{m}\right)\right), \min \left(Z\left(e_{m}\right)\right)\right), \\ \left(\max \left(A\left(e_{m}\right)\right), \max \left(S\left(e_{m}\right)\right), \max \left(D\left(e_{m}\right)\right)\right)\end{array}\right\}$ $(m=1,2, \ldots, k)$, then

$$
Q(e)^{-} \leq \operatorname{FNIFWMSM}_{k \xi}^{n}\left(Q\left(e_{q}\right), Q\left(e_{2}\right), \ldots, Q\left(e_{k}\right)\right) \leq Q(e)^{+} .
$$

Property 8 (commutativity). Let $Q\left(e_{m}\right)(m=1,2, \ldots, k)$ be a set of FNIFNs and $Q\left(e_{m}^{\prime}\right)(m=1,2, \ldots, k)$ be any permutation of $Q\left(e_{m}\right)(m=1,2, \ldots, k)$, then

$$
\begin{aligned}
& \operatorname{FNIFWMSM}_{k \xi}^{n}\left(Q\left(e_{1}\right), Q\left(e_{2}\right), \ldots, Q\left(e_{m}\right)\right) \\
& \quad=\operatorname{FNIFWMSM}_{k \xi}^{n}\left(Q\left(e_{1}^{\prime}\right), Q\left(e_{2}^{\prime}\right), \ldots,\left(e_{m}^{\prime}\right)\right) .
\end{aligned}
$$

\section{Numerical Example}

The quality of higher education has always been an important issue of general concern. It is also a hot issue that has been widely concerned by all sectors of society since the popularization of higher education in China. At the end of the last century, colleges and universities began to expand the enrollment scale beyond the norm, among which the enrollment of higher vocational colleges increased the most. According to the national long-term development plan and outline for education in 2010-2020, by 2020, the number of students in higher vocational colleges should account for nearly half of the total number of students of all universities and colleges. The education of higher vocational colleges has become a key link in the system of cultivating talents in today's society, and its importance is self-evident. With the rapid growth of enrollment in higher vocational colleges, the hardware facilities well equipped and the urgency of improving teaching quality is becoming increasingly prominent. The Ministry of Education has also issued a series of documents requiring higher vocational colleges to strengthen the quality awareness, especially to strengthen the construction of the teaching quality evaluation system of all disciplines in higher vocational colleges. At the same time, due to the stable and even reduced number of new high school graduates in recent years, general colleges and higher vocational colleges have launched fierce competition in enrollment. If higher vocational colleges want to survive and develop, they must attach great importance to the teaching quality, the construction of teaching staff, the creation of a good learning atmosphere for students, and the improvement of vocational skills. With innovative education concept, higher vocational colleges should improve the system construction of all institutions of the college, build a teaching mechanism with social demand as the guide, employment as the goal, and training high-quality and high skilled vocational talents as the responsibility, and establish the development direction for colleges as well. English teaching plays an indispensable role in higher vocational education. In recent years, various relevant documents issued by the Ministry of Education have mentioned the urgency and importance of colleges and universities for the cultivation of high-quality, multiskilled international talents. With the deep promotion of global economic integration, talents who master English pragmatic ability and are familiar with professional English and professional skills have shown great advantages in learning advanced foreign knowledge and understanding advanced foreign concepts and skills and other international aspects. It can be seen that the English education of higher vocational colleges is undertaking more and more responsibilities for cultivating high-quality and high skilled talents in the new era and new development environment, and the improvement of the English teaching quality in higher vocational colleges requires the improvement of the teaching quality evaluation system to better monitor and guide. A point in case about the selection of the excellent college English teachers with FNIFNs would be utilized to illustrate the above methods. We shall give 5 college English teachers $H_{i}(i=1,2,3,4,5)$ to choose. The experts select four attributes to evaluate these college English teachers: (1) $J_{1}$ represents teaching content; (2) $J_{2}$ means teachers' specialization degree; (3) $J_{3}$ represents teachers' artistic accomplishment; and (4) $J_{4}$ means the teachers' appreciation ability. Several college English teachers shall be depicted with FNIFNs by the DMs on the strength of 4 criterions (whose weighting vector $\xi=(0.15,0.40,0.25,0.20))$; the FNIFN decision matrix is depicted in Table 1.

Then, we shall use the developed method to select the excellent college English teachers.

Step 1. According to Table 1, we can fuse all FNIFNs $r_{i j}$ by FNIFWMSM; use $U=(1,2,3,4)$ and 
TABle 1: The FNIFN DM.

\begin{tabular}{ccccc}
\hline & $J_{1}$ & $J_{2}$ & $J_{3}$ & $J_{4}$ \\
\hline$H_{1}$ & $\langle(0.1,0.2,0.3),(0.2,0.5,0.6)\rangle$ & $\langle(0.2,0.3,0.3),(0.2,0.5,0.5)\rangle$ & $\langle(0.4,0.5,0.5),(0.3,0.4,0.4)\rangle$ & $\langle(0.2,0.3,0.3),(0.4,0.4,0.6)\rangle$ \\
$H_{2}$ & $\langle(0.5,0.7,0.7),(0.1,0.2,0.2)\rangle$ & $\langle(0.3,0.4,0.5),(0.1,0.2,0.4)\rangle$ & $\langle(0.4,0.4,0.6),(0.2,0.2,0.3)\rangle$ & $\langle(0.5,0.6,0.6),(0.2,0.3,0.3)\rangle$ \\
$H_{3}$ & $\langle(0.4,0.5,0.6),(0.2,0.3,0.3)\rangle$ & $\langle(0.6,0.6,0.7),(0.1,0.1,0.2)\rangle$ & $\langle(0.5,0.6,0.6),(0.1,0.2,0.2)\rangle$ & $\langle(0.6,0.6,0.7),(0.1,0.1,0.2)\rangle$ \\
$H_{4}$ & $\langle(0.2,0.2,0.4),(0.3,0.3,0.4)\rangle$ & $\langle(0.1,0.3,0.4),(0.2,0.3,0.5)\rangle$ & $\langle(0.4,0.6,0.6),(0.1,0.2,0.3)\rangle$ & $\langle(0.1,0.4,0.5),(0.2,0.3,0.4)\rangle$ \\
$H_{5}$ & $\langle(0.1,0.1,0.4),(0.3,0.3,0.4)\rangle$ & $\langle(0.4,0.5,0.5),(0.3,0.3,0.4)\rangle$ & $\langle(0.3,0.4,0.4),(0.4,0.5,0.5)\rangle$ & $\langle(0.2,0.2,0.4),(0.1,0.2,0.2)\rangle$ \\
\hline
\end{tabular}

TABLE 2: The coalesced values by the FNIFWMSM operators.

\begin{tabular}{lcc}
\hline Aggregation operator & College English teachers & Operation results \\
\hline & $H_{1}$ & $<(0.2207,0.3191,0.3385),(0.3055,0.4679,0.5362)>$ \\
FNIFWMSM & $H_{2}$ & $<(0.3999,0.4978,0.5760),(0.1830,0.2536,0.3227)>$ \\
& $H_{3}$ & $<(0.5107,0.5571,0.6255),(0.1795,0.1996,0.2503)>$ \\
& $H_{4}$ & $<(0.1770,0.3659,0.4599),(0.2181,0.2979,0.4215)>$ \\
& $H_{5}$ & $<(0.2518,0.3017,0.4110),(0.2902,0.3487,0.3941)>$ \\
\hline
\end{tabular}

TABle 3: The SF of the college English teachers.

\begin{tabular}{lc}
\hline & FNIFWMSM \\
\hline$H_{1}$ & -0.1450 \\
$H_{2}$ & 0.2397 \\
$H_{3}$ & 0.3554 \\
$H_{4}$ & 0.0333 \\
$H_{5}$ & -0.0289 \\
\hline
\end{tabular}

TABLE 4: Order of the college English teachers.

\begin{tabular}{lc}
\hline & Order \\
\hline FNIFWMSM & $H_{3}>H_{2}>H_{4}>H_{5}>H_{1}$ \\
\hline
\end{tabular}

FNIFWDMSM operator to calculate global FNIFNs $H_{i}(i=1,2,3,4,5)$ of the college English teachers $H_{i}$; the coalesced values are shown in Table $2(n=2)$.

Step 2. The SF of college English teachers is calculated in Table 3.

Step 3. According to T3, the order of the college English teachers is depicted in Table 4. Note that ">" means "preferred to." The best college English teachers are $\mathrm{H}_{3}$.

\section{Conclusion}

As we all know, the demand for English majors is obviously on the rise, which puts forward new and higher requirements for application-oriented undergraduate colleges to train compound English majors. However, from the perspective of teaching quality evaluation of English majors in application-oriented undergraduate colleges, the results are not optimistic. Therefore, it is an important task for higher education research in China to explore the problems existing in the process of teaching quality evaluation for English majors in application-oriented undergraduate colleges and how to better train qualified and versatile talents for English majors to adapt to the economic and social development in the new era. We study the MADM issues with FNIFNs and utilize the MSM formula to build several MSM fused formulas with FNIFNs: FNIFMSM operator and FNIFWMSM formula. The characteristic of these two operators is also deliberated. The FNIFWMSM formula is utilized to cope with the MADM issues with FNIFNs. Finally, a point in case for English teaching quality evaluation is employed to depict the raised method. Later, the adhibition and expansion of the built formulas of FNIFNs would be debated in the other MADM direction [49-56].

\section{Data Availability}

The data used to support the findings of this study are included in the article.

\section{Conflicts of Interest}

The author declares that there are no conflicts of interest.

\section{References}

[1] L. A. Zadeh, "Fuzzy sets," Information and Control, vol. 8, no. 3, pp. 338-353, 1965.

[2] H. G. Çitil, "Important notes for a fuzzy boundary value problem," Applied Mathematics and Nonlinear Sciences, vol. 4, no. 2, pp. 305-314, 2019.

[3] H. G. Çitil, "Investigation of a fuzzy problem by the fuzzy laplace transform," Applied Mathematics and Nonlinear Sciences, vol. 4, no. 2, pp. 407-416, 2019.

[4] N. M. Lanbaran, E. Celik, and M. Yiğider, "Evaluation of investment opportunities with interval-valued fuzzy topsis method," Applied Mathematics and Nonlinear Sciences, vol. 5, no. 1, pp. 461-474, 2020.

[5] F. B. Benli, O. A. İlhan, and Ö. Keskin, "An allee threshold model for a glioblastoma (GB)-immune system (IS) interaction with fuzzy initial values," Applied Mathematics and Nonlinear Sciences, vol. 5, no. 1, pp. 499-508, 2019.

[6] F. Meng, J. Tang, and H. Fujita, "Linguistic intuitionistic fuzzy preference relations and their application to multi-criteria decision making," Information Fusion, vol. 46, pp. 77-90, 2019. 
[7] Z. Liu and F. Xiao, "An evidential aggregation method of intuitionistic fuzzy sets based on belief entropy," IEEE Access, vol. 7, pp. 68905-68916, 2019.

[8] K. T. Atanassov, "Intuitionistic fuzzy sets," Fuzzy Sets and Systems, vol. 20, no. 1, pp. 87-96, 1986.

[9] K. T. Atanassov, "More on intuitionistic fuzzy sets," Fuzzy Sets and Systems, vol. 33, no. 1, pp. 37-45, 1989.

[10] S. Zeng, W. Su, and L. Sun, "A method based on similarity measures for interactive group decision-making with intuitionistic fuzzy preference relations," Applied Mathematical Modelling, vol. 37, no. 10-11, pp. 6909-6917, 2013.

[11] F. Lei, G. Wei, and X. Chen, "Model-based evaluation for online shopping platform with probabilistic double hierarchy linguistic CODAS method," International Journal of Intelligent Systems, vol. 36, no. 9, pp. 5339-5358, 2021.

[12] G. Wei, J. Wu, Y. Guo, J. Wang, and C. Wei, "An extended COPRAS model for multiple attribute group decision making based on single-valued neutrosophic 2-tuple linguistic environment," Technological and Economic Development of Economy, vol. 27, no. 2, pp. 353-368, 2021.

[13] N. Liao, G. Wei, and X. Chen, "TODIM method based on cumulative prospect theory for multiple attributes group decision making under probabilistic hesitant fuzzy setting," International Journal of Fuzzy Systems, 2021, in press.

[14] H. Zhang, G. Wei, and X. Chen, "CPT-MABAC method for spherical fuzzy multiple attribute group decision making and its application to green supplier selection," Journal of Intelligent \& Fuzzy Systems, vol. 41, no. 1, pp. 1009-1019, 2021.

[15] S. Zhang, H. Gao, G. Wei, and X. Chen, "Grey relational analysis method based on cumulative prospect theory for intuitionistic fuzzy multi-attribute group decision making," Journal of Intelligent \& Fuzzy Systems, vol. 41, no. 2, pp. 3783-3795, 2021.

[16] X. Li and X. Chen, "D-intuitionistic hesitant fuzzy sets and their application in multiple attribute decision making," Cognitive Computation, vol. 10, no. 3, pp. 496-505, 2018.

[17] R. Joshi, S. Kumar, D. Gupta, and H. Kaur, "A jensen- $\alpha$-norm dissimilarity measure for intuitionistic fuzzy sets and its applications in multiple attribute decision making," International Journal of Fuzzy Systems, vol. 20, no. 4, pp. 11881202, 2018.

[18] D. K. Iakovidis and E. Papageorgiou, "Intuitionistic fuzzy cognitive maps for medical decision making," IEEE Transactions on Information Technology in Biomedicine, vol. 15, no. 1, pp. 100-107, 2011.

[19] D.-F. Li, "The GOWA operator based approach to multiattribute decision making using intuitionistic fuzzy sets," Mathematical and Computer Modelling, vol. 53, no. 5-6, pp. 1182-1196, 2011.

[20] Z. X. Su, M. Y. Chen, G. P. Xia, and L. Wang, "An interactive method for dynamic intuitionistic fuzzy multi-attribute group decision making," Expert Systems with Applications, vol. 38, no. 12, pp. 15286-15295, 2011.

[21] C. Tan, "A multi-criteria interval-valued intuitionistic fuzzy group decision making with choquet integral-based TOPSIS," Expert Systems with Applications, vol. 38, no. 4, pp. 30233033, 2011.

[22] J.-Z. Wu and Q. Zhang, "Multicriteria decision making method based on intuitionistic fuzzy weighted entropy," Expert Systems with Applications, vol. 38, no. 1, pp. 916-922, 2011.

[23] D. Yu, "Group decision making based on generalized intuitionistic fuzzy prioritized geometric operator," International Journal of Intelligent Systems, vol. 27, no. 7, pp. 635-661, 2012.
[24] S. Yu, Z. S. Xu, and S. S. Liu, "Derivatives and differentials for multiplicative intuitionistic fuzzy information," Applied Mathematics-A Journal of Chinese Universities, vol. 32, no. 4, pp. 443-461, 2017.

[25] X. Zhao, Y. Zheng, and Z. Wan, "Interactive intuitionistic fuzzy methods for multilevel programming problems," Expert Systems with Applications, vol. 72, pp. 258-268, 2017.

[26] A. Arya and S. P. Yadav, "Development of intuitionistic fuzzy super-efficiency slack based measure with an application to health sector," Computers \& Industrial Engineering, vol. 115, pp. 368-380, 2018.

[27] G. Büyüközkan, O. Feyzioğlu, and F. Göçer, "Selection of sustainable urban transportation alternatives using an integrated intuitionistic fuzzy choquet integral approach," Transportation Research Part D: Transport and Environment, vol. 58, pp. 186-207, 2018.

[28] S. K. De and S. S. Sana, "The $(p, q, r, l)$ model for stochastic demand under intuitionistic fuzzy aggregation with bonferroni mean," Journal of Intelligent Manufacturing, vol. 29, no. 8, pp. 1753-1771, 2018.

[29] H. Garg, "An improved cosine similarity measure for intuitionistic fuzzy sets and their applications to decision-making process," Hacettepe Journal of Mathematics and Statistics, vol. 47, pp. 1578-1594, 2018.

[30] J. Q. Li, W. Chen, Z. L. Yang, and C. Y. Li, "A time-preference and VIKOR-based dynamic intuitionistic fuzzy decision making method," Filomat, vol. 32, no. 5, pp. 1523-1533, 2018.

[31] M. Zhao, G. Wei, X. Chen, and Y. Wei, "Intuitionistic fuzzy MABAC method based on cumulative prospect theory for multiple attribute group decision making," International Journal of Intelligent Systems, vol. 36, no. 11, pp. 6337-6359, 2021.

[32] S. Niroomand, "A multi-objective based direct solution approach for linear programming with intuitionistic fuzzy parameters," Journal of Intelligent \& Fuzzy Systems, vol. 35, no. 2, pp. 1923-1934, 2018.

[33] M. Zhao, G. Wei, C. Wei, and J. Wu, "Improved TODIM method for intuitionistic fuzzy MAGDM based on cumulative prospect theory and its application on stock investment selection," International Journal of Machine Learning and Cybernetics, vol. 12, no. 3, pp. 891-901, 2021.

[34] F. Liu and X. H. Yuan, "Fuzzy number intuitionistic fuzzy set," Fuzzy Systems and Mathematics, vol. 21, pp. 88-91, 2007.

[35] J. Li, Q. Niu, and C. Y. Zhang, "Similarity measure and fuzzy entropy of fuzzy number intuitionistic fuzzy sets," in Fuzzy Information and Engineering, B. Y. Cao, C. Y. Zhang, and T. F. Li, Eds., vol. 1, pp. 373-387, Taylor \& Francis, Abingdon, UK, 2009.

[36] X. F. Wang, "Fuzzy number intuitionistic fuzzy geometric aggregation operators and their application to decision making," Control and Decision, vol. 23, pp. 607-612, 2008.

[37] R. Verma, "Generalized Bonferroni mean operator for fuzzy number intuitionistic fuzzy sets and its application to multiattribute decision making," International Journal of Intelligent Systems, vol. 30, no. 5, pp. 499-519, 2015.

[38] H.-C. Liu, M.-Y. Quan, Z. Li, and Z.-L. Wang, "A new integrated MCDM model for sustainable supplier selection under interval-valued intuitionistic uncertain linguistic environment," Information Sciences, vol. 486, pp. 254-270, 2019.

[39] W. Y. Yu, Z. Zhang, and Q. Y. Zhong, "Consensus reaching for MAGDM with multi-granular hesitant fuzzy linguistic term sets: a minimum adjustment-based approach," Annals of Operations Research, vol. 300, 2020. 
[40] Y. Huang, R. Lin, and X. Chen, "An enhancement EDAS method based on prospect theory," Technological and Economic Development of Economy, vol. 27, no. 5, pp. 1019-1038, 2021.

[41] T. He, G. Wei, J. Wu, and C. Wei, "Qualiflex method for evaluating human factors in construction project management with pythagorean 2-tuple linguistic information," Journal of Intelligent \& Fuzzy Systems, vol. 40, no. 3, pp. 4039-4050, 2021.

[42] Z. Li, G. Wei, and M. Lu, "Pythagorean fuzzy hamy mean operators in multiple attribute group decision making and their application to supplier selection," Symmetry, vol. 10, no. 10, p. 505, 2018.

[43] T. Hara, M. Uchiyama, and S. E. Takahasi, "A refinement of various mean inequalities," Journal of Inequalities and Applications, vol. 2, pp. 387-395, 1998.

[44] C. Maclaurin, "IV a second letter from Mr. Colin McLaurin, professor of mathematicks in the university of Edinburgh and F. R. S. to Martin Folkes, esq; concerning the roots of equations, with the demonstration of other rules in algebra; being the continuation of the letter published in the philosophical transactions, N 394 ," Philosophical Transactions, vol. 36, pp. 59-96, 1730.

[45] P. Liu, H. Yang, H. Wu, M. Ju, and F. E. Alsaadi, "Some maclaurin symmetric mean aggregation operators based on cloud model and their application to decision-making," International Journal of Information Technology \& Decision Making, vol. 18, no. 3, pp. 981-1007, 2019.

[46] P. Liu, Y. Li, and M. Zhang, "Some Maclaurin symmetric mean aggregation operators based on two-dimensional uncertain linguistic information and their application to decision making," Neural Computing and Applications, vol. 31, no. 8, pp. 4305-4318, 2019.

[47] H. Garg, "Hesitant pythagorean fuzzy maclaurin symmetric mean operators and its applications to multiattribute decision-making process," International Journal of Intelligent Systems, vol. 34, no. 4, pp. 601-626, 2019.

[48] X. F. Wang, "Fuzzy number intuitionistic fuzzy arithmetic aggregation operators," International Journal of Fuzzy Systems, vol. 10, pp. 104-111, 2008.

[49] A. P. Darko and D. C. Liang, "Some q-rung orthopair fuzzy hamacher aggregation operators and their application to multiple attribute group decision making with modified EDAS method," Engineering Applications of Artificial Intelligence, vol. 87, 2020.

[50] G. Sirbiladze, “Associated probabilities' aggregations in interactive multiattribute decision making for $q$-rung orthopair fuzzy discrimination environment," International Journal of Intelligent Systems, vol. 35, no. 3, pp. 335-372, 2020.

[51] J. Chai, S. Xian, and S. Lu, "Z-uncertain probabilistic linguistic variables and its application in emergency decision making for treatment of COVID-19 patients," International Journal of Intelligent Systems, vol. 36, no. 1, pp. 362-402, 2021.

[52] D. C. Liang, Z. Y. Dai, and M. W. Wang, "Assessing customer satisfaction of $\mathrm{O} 2 \mathrm{O}$ takeaway based on online reviews by integrating fuzzy comprehensive evaluation with AHP and probabilistic linguistic term sets," Applied Soft Computing, vol. 98, 2021.

[53] P. Liu and P. Wang, "Some q-rung orthopair fuzzy aggregation operators and their applications to multiple-attribute decision making," International Journal of Intelligent Systems, vol. 33, no. 2, pp. 259-280, 2018.

[54] P. Liu and X. Zhang, "A novel picture fuzzy linguistic aggregation operator and its application to group decision- making," Cognitive Computation, vol. 10, no. 2, pp. 242-259, 2018.

[55] D. Li, Y. Wang, A. Madden et al., "Analyzing stock market trends using social media user moods and social influence," Journal of the Association for Information Science and Technology, vol. 70, no. 9, pp. 1000-1013, 2019.

[56] L. Jiang, T. Zhang, and Y. Feng, "Identifying the critical factors of sustainable manufacturing using the fuzzy dematel method," Applied Mathematics and Nonlinear Sciences, vol. 5, no. 2, pp. 391-404, 2020. 